\title{
Gesundheit und Gesundheitsverhalten im Jugendalter. Der Einfluss sozialer Ungleichheit.
}

Wer sich mit dem Thema Gesundheit von Jugendlichen befasst, begegnet immer wieder der internationalen Studie „Health Behaviour in School-aged Children (HBSC)“. Nun liegt eine neue bemerkenswerte Arbeit basierend auf deutschen Daten des Forschungsnetzes vor, welche Gesundheit und Gesundheitsverhalten von 11- bis 15-Jährigen in Abhängigkeit von sozialer Ungleichheit zum Thema macht. Matthias Richter geht dieses Vorhaben fundiert, detailliert, systematisch und gut verständlich an.

Die erste Hälfte des Buches ist dem theoretischen und empirischen Hintergrund des Themas gewidmet, anhand dessen die Fragestellungen der Studie entwickelt werden. Zunächst wird der Begriff der sozialen Ungleichheit geklärt und die Problematik ihrer Erfassung kritisch beleuchtet. Dies wird ergänzt durch einen kurzen Abriss der Diskussionen um das Konzept der sozialen Schichtung.

Das nächste Kapitel zeigt einmal mehr eindrücklich die Vielfalt der Befunde zu sozioökonomischen Unterschieden in Mortalität, Morbidität und gesundheitsrelevantem Verhalten bei Erwachsenen.

Danach wendet sich Richter den bekannten Ergebnissen zu sozialer Ungleichheit und Gesundheit im Jugendalter zu. Der Autor erhebt dabei keinen Anspruch auf Vollständigkeit der zitierten Studien, bietet aber ein breites Spektrum an Ergebnissen. Diese belegen nur wenige Zusammenhänge zwischen sozialer Ungleichheit und Gesundheit und Gesundheitsverhalten in diesem Alter. Teilweise sind die Zusammenhänge sogar entgegen der generellen Erwartung ungünstiger für Jugendliche mit höherem sozioökonomischem Status (SES). Insgesamt sind die Ergebnisse inkonsistent, was teilweise inhaltlich begründbar, teilweise auch auf begriffliche und methodische Unterschiede zurückzuführen ist. Verschiedene Probleme betreffen die Vergleichbarkeit der untersuchten Altersgruppen, aber auch der Definitionen und Operationalisierungen von sozialer Ungleichheit bzw. sozioökonomischem
Status sowie Gesundheit und Gesundheitsverhalten. Auffällig ist ungeachtet dieser methodischen Schwierigkeiten, dass bei Kindern und Erwachsenen soziale Ungleichheiten in der Gesundheit festgestellt werden, dass solche Unterschiede bei Jugendlichen aber seltener und weniger deutlich sind.

Im letzten theoretischen Kapitel des Buches werden vier Erklärungsansätze für soziale Ungleichheit in der Gesundheit von Erwachsenen systematisch aufgearbeitet und überschaubar dargestellt. Obwohl diese Thesen grundsätzlich auch für Jugendliche diskutiert werden können, sind darüber hinaus jugendspezifische Erklärungsansätze sinnvoll. Diese beschäftigen sich vor allem mit der Frage, weshalb bei Jugendlichen weniger soziale Ungleichheiten im Bereich der Gesundheit festgestellt werden:

Die Hypothese latenter Unterschiede geht davon aus, dass Jugendliche noch relativ gesund sind und deshalb keine sozialen Ungleichheiten für Mortalität und Morbidität gefunden werden können. Die Grundlagen für unterschiedliche Gesundheitsbeeinträchtigungen im Erwachsenenalter sind aber laut dieser Hypothese bei Jugendlichen schon gelegt. Deshalb sollten sich für früher sichtbare gesundheitliche Beschwerden und Beeinträchtigungen, aber auch für Gesundheitsverhalten bereits in diesem Alter soziale Ungleichheiten zeigen.

Die Pufferhypothese hingegen basiert auf der Annahme, dass Gleichaltrige und die Schule soziale Unterschiede im Jugendalter ausgleichen. Dies wird dadurch begünstigt, dass der sozioökonomische Status der Eltern für die Jugendlichen mit zunehmendem Alter an Aussagekraft verliert, der eigene SES aber noch nicht richtig ausgebildet ist.

Der empirische Teil der Arbeit beginnt mit einer detaillierten Darstellung der verwendeten Variablen und einer Beschreibung der durchgeführten Analysen. Diese Ausführlichkeit erscheint für die Gewährleistung der interdisziplinären Verständigung in Public Health sehr sinnvoll und nachahmenswert. 
Die Analysen der repräsentativen Daten von 11- bis 15jährigen Jugendlichen in Deutschland bieten detaillierte Ergebnisse zu inhaltlich ganz verschiedenen Indikatoren des Gesundheitsstatus und Gesundheitsverhaltens. Alters- und Geschlechtsunterschiede werden dabei systematisch mitberücksichtigt, so dass Zusammenhänge zwischen SES und Gesundheit für die entsprechenden Gruppen differenziert werden können.

Bei der Beschreibung der gesundheitlichen Lage der Jugendlichen stellt Richter fest, dass die Beeinträchtigungen bereits in diesem Alter bedeutender sind als im Allgemeinen angenommen wird.

Der Einfluss der beiden SES-Indikatoren Berufsstatus der Eltern und familiärer Wohlstand auf Gesundheit und gesundheitliche Beeinträchtigungen ist zum Teil deutlich, geht aber, in Übereinstimmung mit bisher bekannten Befunden, nicht immer in die gleiche Richtung. Herauszuheben sind das häufigere Übergewicht bei Jugendlichen mit niedrigem SES und die häufigeren Allergien bei Jugendlichen mit hohem SES.

Mit der gleichen Systematik werden Ergebnisse zum Gesundheitsverhalten dargestellt. Mit zunehmendem Alter wird das gesundheitsrelevante Verhalten generell ungünstiger. Gleichzeitig differenzieren sich für verschiedene Verhaltensweisen soziale Unterschiede heraus, allerdings auch hier wieder in unterschiedlicher Richtung. Zeigen Indikatoren zum Ernährungsverhalten, zu Fernsehkonsum und Rauchen ungünstigere Verhaltensmuster bei den Jugendlichen mit niedrigem SES, finden sich zum Alkoholkonsum gegenteilige Ergebnisse.

Schliesslich werden Ergebnisse einer aufwändigen Analysereihe präsentiert, welche den Einfluss der Schule und der Gleichaltrigen auf die soziale Ungleichheit bezüglich ausgewählter Variablen der Gesundheit und des Gesundheitsverhaltens untersucht. Es zeigt sich, dass Variablen zu Peers und Schule über den sozioökonomischen Status hinaus bedeutenden zusätzlichen Erklärungswert haben oder sogar zwischen sozialer Ungleichheit und Gesundheit und Gesundheitsverhalten vermitteln. Die Ergebnisse lassen nicht abschliessend über das Zutreffen der Pufferhypothese entscheiden, weisen aber deutlich auf die wichtige Rolle der Gleichaltrigen und der Schule in diesem Alter hin.

Zur Überprüfung dieser Hypothese wurde der Einfluss von Peers und Schule auf Gesundheitsindikatoren untersucht, bei welchen sich in der vorangehenden bivariaten Analyse eine soziale Ungleichheit zeigte. Interessant wäre als weitere Untersuchung der Pufferhypothese, ob und in welchem Ausmass Peers und Schule eine vermittelnde Rolle spielen für Indikatoren, bei denen im ersten Schritt keine sozioökonomischen Unterschiede feststellbar waren.

Anregend für weitere Forschungsfragen sind auch Richters weiterführende Überlegungen zu verschiedenen Ergebnissen, etwa dazu, weshalb bei Mädchen grössere Effekte sozialer Ungleichheit festzustellen sind als bei Jungen, während der Effekt bei erwachsenen Frauen geringer ist als bei Männern. Eine detaillierteZusammenfassung mit hilfreichen Übersichtstabellen und eine ausführliche Diskussion der Ergebnisse auch im Hinblick auf praktische Implikationen runden die Arbeit ab. Damit kann das Buch sowohl für einen aktuellen Überblick zum Thema als auch zum Nachschlagen spezifischer Ergebnisse empfohlen werden.

\section{Corinna Bisegger, Bern e-mail: bisegger@ispm.unibe.ch}

